Rev, Elev. Méd. vét. Pays trop., 1968, 21, 4 (493-498).

\title{
Hémolymphe de glossines : récolte et analyse
}

\author{
por J. P. PETIT \\ Laboratoire de Biochımie de I'I. E. M. V. T.
}

\begin{abstract}
RÉSUME
En vue de cultiver des souches de trypanosomes pathogènes sur cellules de glossines, l'étude d'un milieu pour cultures cellulaires de composition voisine de l'hémolymphe est entreprise.

Les analyses nécessaıres à la détermination exacte des normes biochimiques et des composants de l'hémolymphe nécessitant des quantités relativement élevées d'échantillons, les divers protocoles de récolte sont étudiés et critiqués en fonction de l'explotation finale des résultats.

Les prélèvements sont faits dans les élevages de $G$. austeni, G. tachinoides et G. morsitons morsitons.

Les analyses ont porté sur le $\mathrm{pH}$, la pressıon osmotique des éléments chimiques classiques et les acides aminés libres.

Le pH de l'hémolymphe se situe autour de 6,8 et coincide de façon remarquable avec celui des milieux de cultures cellulaires déjà essayés par d'autres auteurs.

Les résultats obtenus autorisent la poursuite des essais.
\end{abstract}

\section{INTRODUCTION}

- La culture des trypanosomes pathogènes a déjà fait l'objet de très nombreuses récherches et la multiplicité des travaux publiés à ce sujet, si elie prouve l'importance qu'on y attache, montre également qu'aucune des solutions proposées jusqu'à maıntenant ne s'est avérée satisfaisante. On peut résumer la situation en disant que des succès ont été obtenus puisque les parasites ont été maintenus en survie et même parfois se sont multipliés, mais il a encore été impossible de cultiver systématıquement des trypanosomes avec conservation de leurs propriétés originelles pathogènes. Dans un rapport, ITARD (J.) (7) écrit qu'un milieu de culture ne devait être, en princıpe, composé qu'après une analyse poussée de l'hémolymphe de l'insecte considérë.

C'est pourquoi le problème a été abordé sous un angle différent en partant du fait que I'Ins- fitut dispose d'un élevage de glossines en pleine. expansion, ce qui représente un matériel de base pour les analyses.

- L'idée générale n'est pas nouvelle puisque TRAGER (W). (10) s'en inspirait déjà en 1959 dans une étude extrêmement poussée; elle a été reprise depuis par NICOLI (J.) et VATIER ( 9 ) qui ne pouvaient que constater le bilan négatif de ces essais ef essayer de l'expliquer. On doit chercher à connaître la composition chimique de l'hémolymphe pour reconstituer artificiellelement ce milieu et y cultiver des cellules de glossines. On peut penser qu'ensuite il sera possible d'y entretenir des souches de trypanosomes pathogènes pour les animaux, ou pour l'homme.

- Ce premier travail vise à étudier le mode de prélèvement de l'hémolymphe ef à donner les. résultats des premières analyses effectuées sur les échantillons ainsi récoltés. II ne s'agit pas de 
faire l'historique des cultures de cellules d'invertébrés pour lesquelles un des chercheurs les plus avancés actuellement est le Docteur VAGO, encore moins celui des cultures de trypanosomes.

\section{I. - MODES DE PRÉLÈVEMENT}

Selon le but que l'on se propose d'atteindre on peut retenir 4 méthodes principales qui peuvent se classer en deux grands groupes :

$1^{\circ}$ La méthode intégrale qui s'adresse à des populations : glossines totales ou pupes broyées, portions de glossines broyées.

$2^{\circ}$ La méthode sélective qui s'adresse à des individus : méthode du piston liquide, méthode par exsudation.

\section{Méthode intégrale.}

a) extrats totaux.

- On broie ensemble une quantité importante d'insectes ou de pupes pour en extraire ensulte un liquide brut par centrifugation. Quand on voudra procéder à des analyses, on réunira plusieurs extraits pour obtenir la quantité d'échantillons nécessaire. En général on constitue des lots sur environ $2 \mathrm{ml}$, ce qui correspond à un nombre de 450 à 800 individus selon les espèces de glossınes. Les résultats obtenus constitueront une bonne approximation du taux moyen de tel ou tel élément chez une glossine. Cette méthode permet de récolter les extraits au fur et à mesure de la production de l'élevage et, en s'adressant à des mouches d'âge détermıné, de rapporter les résultats à un stade donné du cycle biologique.

- Pour des raisons de commadité de manipulation nous avons utilisé principalement dans ce travail des pupes prêtes à éclore, considérant comme hypothèse d'approche que leur composition serait assez voisine de celle de mouches venant de sortir des pupes. La récolte est ainsi rapide et permet d'aboutir relativement vite à des quantités d'extrait analysables. Ce procédé n'a pas que des avantages, en particulier certaines réserves s'imposent quant aux différences qui peuvent exister entre l'hémolymphe circulante de l'insecte ef l'extrait brut total obtenu par centrifugation après broyage. Un autre inconvénıent réside dans le fait qu'on étudie en un mélange indistinct les deux sexes, mais on peut $y$ pallier en utilisant des mouches frâchement écloses.

b) extroits partiels.

- Le procédé est voisin du précédent, mais il s'adresse uniquement aux mouches et le broyage n'est fait qu'après les avar séparées en leurs diverses parties : tête, thorax, pattes, abdomen, etc... Les renselgnements obtenus après dosages, se rapporteront aınsi aux éléments disséqués avant l'obtention de l'extrait liquide brut. La finesse de l'analyse est icı en rapport direct avec la finesse de la dissection.

\section{Méthode sélective.}

a) méthode du piston liquide.

- Extrêmement séduisant, le procédé consiste à réaliser deux perforations dans la chitine de l'insecte, l'une sous l'abdomen, l'autre sur le côté du thorox, puis à injecter du liquide de Ringer au 1/10 par le thorax; on récolte la goutte de liquide qui perle au niveau de l'abdomen. On utilise une ultramicro-seringue capable de mesurer le demi-microlitre et des aiguilles capillaires.

- L'hypothèse de base est que le liquide injecté refoule dans l'insecte l'hémolymphe circulante sans s'y mélanger, sauf dans la zone de contact, qui n'est pas récoltée: on ne recueılle que la première goutte qui perle après avorr injecté seulement de 1 à 2 microlitres dans le thorax: WYATT ( S. S.) (11) a déjà récolté ainsi l'hémolymphe du ver à soie et GRACE (T. D. C.) celle de Callosamia (5) et du même ver à soie (6) ; ces auteurs ont travaillé chez la larve et ont été gênés par la grande quantité de lipides présents.

- Le principal avantage de ce mode de récolte est l'obtention d'hémolymphe particulièrement pure et le fait que la même mouche peut servir à plusieurs prélèvements successifs. Mais sa pratique est longue et délicate et si le résultat des analyses sur cette hémolymphe est sans critique du point de vue qualitatif on verra qu'il n'en est pas de même au point de vue quantitatif, une erreur due à la dilution étant probable. 


\section{b) méthode de l'exsudation.}

- Extrêmement utilısée en particulier par KNIGHT (R. H.) (8) et BURSELL (A.) (1) pour l'analyse des acides aminés libres; il suffit de sectionner une des pattes postérieures près du corps et de récoiter l'hémolymphe qui exsude dans un tube capillaire appliqué sur la section. En serrant convenablement l'insecte on peut recuelllir jusqu'à 2 microlıtres de liquide mais parfois on ne récolte rien du tout et sur les mouches d'élevage, il a jusqu'ici été impossible de récolter plus de 0,25 microlitres par ce procédé dans nos laboratores.

- Sur les plans théorique et pratique l'hémoSymphe ainsi obtenue est bien le liquide intérieur à l'organisme de l'insecte et les analyses effectuées peuvent être quantitatives, mais la lenteur de la récolte et le peu de liquide collecté ne permettent pas d'obtenir rapidement tous les résultats souhaltés. De plus, elle ne peut pas être pratiquée sur les pupes. Pour l'étude chromatographique de I'hémolymphe ce procédé reste le meilleur car les analyses peuvent alors être pratiquées sur une seule glossıne même avec Ies faibles quantıtés que nous avons pu recueıllir aınsi.

- Pour ce travail les deux modes de prélèvement ont été utilisés, intégral et sélectif, le premier sur les pupes et le second au moyen du piston liquide sur les adultes, ceci en raison du but poursuivi qui étaıt l'analyse rapide d'échantilions suffisamment abondants (broyage) et le contrôle qualitatif de ces analyses par comparaison avec l'hémolymphe récoltée même diluée, par la méthade sélective (pıston lıquide).

\section{II. - MATÉRIEL ET MÉTHODES}

\section{Les glossines.}

L'élevage du Service d'Entomologie de H'I. E. M. V. T. produit régulièrement Glossina austeni, Glossina tachinoides et Glossina morsitans morsitons cette dernière d'origine Rhodésienne. Des pupes de ces trois espèces sont broyées au $24^{e}$ jour de puppaison.

Les mouches sont réparties en jeunes mâles et jeunes femelles, non nourris, à l'éclosion, et vieux mâles à jeun depuis $12 \mathrm{~h}$, ayant servi à la reproduction.

\section{$2^{0}$ Mode d'obtention des prélèvements.}

a) extrait brut des pupes.

On centrifuge à $25.000 \mathrm{~g}$ pendant $55 \mathrm{mn}$ des pupes écrasées avec un agitateur sur un disque en verre fritté $n^{\circ} 00$ soudé dans la lumière d'un tube qui est introduit dans les godets en plastique de la centrifugeuse. Après pipetage du liquide qui a filtré, on centrifuge à nouveau à la même vitesse mais pendant $15 \mathrm{mn}$ seulement et après avoir remalaxé le broyat. On obtient aınsi trois couches superposées nettement distinctes. En surface quelques lipides accompagnés d'une fine pellicule pigmentaire, l'extrait brut liquide et au fond un culat très pigmenté en rouge brun. Ces trois couches sont récoltées séparément en commençant par celle du milieu après avoir incliné le tube pour que les couches extrêmes ne se mélangent pas. On obtient ainsi en moyenne 3.7 microlitres pour Gl. marsitans, 3,5 pour Gl. austeni et 3,6 pour $G$ l. tachinoides. Après la récolte tous les prélèvements sont congelés à $-30^{\circ} \mathrm{C}$ sans délais. On diminue ainsi les risques de mélanisation, qui sont importants.

Dès la décongélation pour dosage on ajoute 1 microlitre/ml d'acide ascorbique en solution aqueuse à $1 \mathrm{mg} / \mathrm{ml}$, sauf pour les mesures de $\mathrm{pH}$.

\section{b) hémolymphe de mouches.}

Une certaine quantité d'hémolymphe a été recueillie par la méthode du piston liquide sur des mâles et des femelles adultes; en général Il faut injecter au moins 2 microlitres de Rınger au $1 / 10$ pour obtenir un peu d'hémolymphe. 3u Méthodes d'analyse.

Les méthodes d'analyses sont toutes celles dont les protocoles ne demandalent que de faibles quantités d'échantıllon ef pour les autres qui ne se trouvaient pas classiquement dans la littérature une miniaturısation a été effectuée au laboratoire. Les protocoles de dosages annsi mis au point feront l'objet de publications séparées. Pour mesurer le $\mathrm{pH}$, un potentiomètre Mefhrohm E 353 a été utilısé et les lectures spectrophotométriques ont été faltes avec un spectrophotomètre PMQ II associé à un enregistreur Sefram GR VAMK.

Il a fallu environ 5.000 mouches de chacune des 3 espèces pour réaliser ce traval. On peut déjà en conclure que les résultats représentent 
une bonne approximation des moyennes rencontrées parmi les populations utilisées.

\section{III. - RÉSULTATS}

- Les principales données physiques qui sont relatives aux pupes prélevés au $24 e$ jour.

permettent de reconstituer un milieu de culture ainsı que le dosage des éléments qu'il semble le plus important de connaître sont regroupés dans le tableau $n^{0} 1$. Toutes les données qu'il contient $N^{\circ}$

Analyses d'extrait brut de pupes de glossines prêlevếes au 24 jou jour.

\begin{tabular}{|c|c|c|c|}
\hline & G. morsitans & G. austeni & G. tachinoides \\
\hline Nombre de pupes & 911 & 1325 & 1255 \\
\hline $\mathrm{pH}$ & 6,65 & 6,62 & 6,62 \\
\hline Azote total en mg/m1 & 7,08 & 6,34 & 13,0 \\
\hline $\begin{array}{c}\text { Pression osmot Ique en } \\
\text { osmoles }\end{array}$ & 0,575 & 0,588 & 0,604 \\
\hline $\begin{array}{c}\text { Phosphore minérral lab1le } \\
\text { en mg p. } 1 \text { O00 }\end{array}$ & 99,6 & 96 & 99,6 \\
\hline $\begin{array}{c}\text { Phosphore total acidosoluble } \\
\text { en mg p. } 1000\end{array}$ & 1220 & 552 & 1880 \\
\hline $\mathrm{Na}^{+} \mathrm{mg} / \mathrm{ml} 1$ & 1,7 & 1,9 & 2,0 \\
\hline $\mathrm{K}^{+} \mathrm{mg} / \mathrm{ml}$ & 1,6 & 2,4 & 2,2 \\
\hline $\mathrm{Na} / \mathrm{K}$ & 1,06 & 0,79 & 0,91 \\
\hline
\end{tabular}

- Des analyses ont pu être pratiquées sur l'hémolymphe récoltée par la méthode du piston liquide sur un mélange de mâles et de femelles de Gl. morsitans, elles figurent dans le tableau no 2 .

\section{TABLEAU $\mathrm{N}^{\circ}$ II}

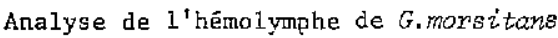
adultes recueillie par la mêthode du piston liquide

\begin{tabular}{|c|c|}
\hline & Hếmoil ymphe de G.morsitans \\
\hline Azote total en mg/ml & 5 \\
\hline $\begin{array}{l}\text { Pression osmotique } \\
\text { en atmosphères }\end{array}$ & 8,0 \\
\hline $\begin{array}{l}\text { Phosphore minéral } \\
\text { mg p. 1000 }\end{array}$ & 30 \\
\hline $\begin{array}{l}\text { Phosphore } \\
\text { acidosoluble } \\
\text { tatal mg p. 1000 }\end{array}$ & 66 \\
\hline $\mathrm{Na}^{+}$en mg/mI & 0,16 \\
\hline $\mathrm{K}^{+}$en mg/mI & 0,25 \\
\hline $\mathrm{Na} / \mathrm{k}$ & 0,64 \\
\hline
\end{tabular}

- Par la méthode électrochromatographique on a pu confirmer la présence d'acides aminés libres dans l'hémolymphe recueillie d̀ la patte postérieure. Les plus abondants étant par ordre décroissant : la proline, l'alanine puis la sérine, la lysine ef l'histidine. Les migrations ont été effectuées sur couche mince de poudre de cellulose MN 300 sans liant. L'électrophorèse a été falte avec le tampon formacétique de $\mathrm{pH}$ 1,25 sous $400 \mathrm{~V}$ et la chromatographie en solvant butanol acide acétique eau (3:1:5). L'hémolymphe étalt déposée directement sur la couche mınce dès après le prélèvement. Avec 0,25 microlitres on peut effectuer une analyse.

\section{IV. - DISCUSSION}

\section{Mesure du $\mathrm{pH}$.}

Le mode de prélèvement semble peu influer sur le $\mathrm{pH}$, mais lors du prélèvement à la seringue de légères différences concernant mâles et femelles ont pu être mises en évidence (tableau no 3 ). Ces résultats montrent l'unifor- 
TABLEAU $\mathrm{N}^{*}$ III

pH de 1 'hếmolymphe de G.morsitcons récoltée, solt à la seringue, soit par centrifugation.

\begin{tabular}{|l|c|c|}
\hline G. moxsitans & $\begin{array}{c}\text { Hémolymphe } \\
\text { à la seringue }\end{array}$ & $\begin{array}{c}\text { Hémolymphe par } \\
\text { centrifugation }\end{array}$ \\
\hline Jeunes femelles & 7,50 & $\begin{array}{c}6,70 \\
\text { (âgées d'1 jour) }\end{array}$ \\
\hline Jeunes mâles & 6,48 & $\begin{array}{c}6,70 \\
\text { (âgés d'l jour) }\end{array}$ \\
\hline Vieux mâles & 6,70 & \\
\hline Pupes 24 j, & & 6,65 \\
\hline
\end{tabular}

misation réalisée au sein de l'extrait brut obtenu par centrifugation que ce soit des pupes ou des mouches, le broyage des tissus libérant sans aucun doute des substances étrangères à l'hémolymphe. Les valeurs les plus intéressantes du $\mathrm{pH}$ sont donc celles relevées sur l'hémolymphe recueillie à la seringue; elles révèlent des différences intéressantes entre les sexes. Une légère variation du $\mathrm{pH}$ au cours de la vie des mâles peut ne correspondre qu'au mode d'alimentation sur lapin ou à une évolution du métabolisme.

- Les meilleurs cultures de TRAGER (10) et de NICOLLI (9) respectivement $D_{4}$ et $D_{2}$ avaient un $\mathrm{pH}$ très voisin de celui de l'hémolymphe : 6,7 à 6,9 et 6,8 . Les essais de culture d'ITARD (7) ont été réalısés avec un milieu de $\mathrm{pH} 6,9$ ce qui semble excellent.

\section{$2^{\circ}$ Analyses.}

Les analyses chimiques résumées dans les tableaux 1 et 2 montrent bien que l'hémolymphe recueililie à la seringue est plus dıluée que l'extrait brut obtenu par centrifugation. Ce seront done les résultats réuns dans le tableau 1 qui serviront de première base à l'élaboration d'un milieu de culture. II n'est malheureusement pas possible de les comparer aux milieux de culture déjà esscyés car il entre dans leur composition de nombreux éléments non synthétiques et les analyses correspondantes sur le milieu total r'ont pas été effectuées sauf pour le milieu essayé par ITARD au laboratoire du Docteur VAGO, qui donne en particulier un rapporf $\mathrm{Na} / \mathrm{K}$, de 23, ce qui semble un peu élevé si on le compare d̀ ceux obtenus (tableau 1).

\section{Acides aminés libres.}

Les acides aminés libres de l'hémolymphe de vieille mouche trouvés en quantité la plus importante sont bien les mêrnes que ceux indiqués par BURSELL (E.) et confirmés par KNIGHT (R. H.) : la proline et l'alanine, en quantité moindre la serine, la lysine n'est trouvée par KNIGHT que dans l'abdomen, tandis que BURSELL en trouve aussı dans l'hémolymphe et que les expériences citées plus haut nous indiquent également son existence; enfin d'histidine non trouvée par ces deux auteurs ef que nous avons rencontrée en faible quantité.

L'étude de ces acides aminés libres demande à être approfondie davantage pour vérifier l'influence du mode d'alimentation et de l'âge des mouches sur eux et rechercher les acides aminés qui seraient en plus faible quantité. L'intérêt de ces composés a été particulièrement démontré par BURSELL qui en a étudié le métabolisme et qui a démontré l'utilisation de la proline comme source d'énergie pour les muscles du vol. Ces particularités liées à des systèmes enzymatiques spéciaux, suffiraient à elles seules à faire engager de nouvelles recherches sur les glossines.

\section{V. - CONCLUSIONS}

Pour essayer d'améliorer le milieu synthétique composé à partir de ces données, sans attendre le résultat des cultures cellulaires, il convient de poursuivre en analysant des éléments dont l'équilibre semble important pour' le métabolisme des insectes, tels que $\mathrm{Ca}^{++}$et $\mathrm{Mg}^{++}$. II importe, particulièrement si les milieux comportent des éléments biologiques non contrôlables, d'effectuer sur eux les mêmes analyses que sur l'hémolymphe afin d'ınstaurer un contrôle réel des milieux.

L'éłude des protéines de l'hémolymphe apportera aussi des connassances importantes à la fois pour le milieu de culture et pour la lutte contre ces hôtes intermédiaires des trypanosomes. Toutes ces recherches sont en cours et s'enchaîneront logiquement à ce travail préliminaire.

Nous remercions particulièrement $M$. ITARD, Chef du Service d'Entomologie pour l'abondance et la régularité de la production en glassines qu'il a bien voulu mettre à notre disposition. 


\section{SUMMARY}

Hemolymphs of tsetseflies : sampling and analysis

In order to cultivate strains of pathogenic trypanosoma on tsetseflies cells. the study of a cellular medium similar to the hemolymph's composition is undertaken.

Relatively large quantities of samples are necessary for exact evaluations of biochemical norms and hemolymph constituants and the different proceedings of collecting are then studied in order to final result exploitation.

The samplings result from breedings of $G$. ousteni, $G$. tochinoides and G. morsitans morsitans.

The analysis have carried out $\mathrm{pH}$, osmotic pressure, classic chemical elements and free amino acid.

The $\mathrm{pH}$ of hemolymph is about to 6,8 and is exactly simılar to this of cellula culture mediums even traying by other authors.

The results then obtained allow previous trials with synthetic culture medium.

\section{RESUMEN}

Hemolinfas de glosinas : recogida y analisis

Para cultivar cepas de tripanosomos patogenos sobre células de glosınas, se estudia un medio para cultivos celulares cuya composición es semejante a la de la hemolinfa.

Necesılan cantidades relativamente elevadas de muestras las analisıs para la determinación exacta de las normas bioquimicas y de los constituyentes de la hemolinfa. Se notan y se critican las varios procedimientos de recogida según la explotación final de los resultados.

Se toman las muestras en las crias de $G$. ousteni, $G$. tachinoides y $G$. morsitans morsitans,

Se analızaron el $\mathrm{pH}$, la presión osmotica de los elementos quimicos clasicos y los ácidos aminados libres

$\mathrm{El} \mathrm{pH}$ de la hemolinfa es de 6,8 poco más o menos y coincide exactamente con el de los medios de cultivos celulares ya experimentados por ofros autores.

Los resultados abtenidos permiten empezar ensayos con un medio de cultivo sıntético.

\section{BIBLIOGRAPHIE ALPHABÉTIQUE}

1. BURSELL (E.). - Free amino acids of the tse tse fly (Glossina). Nature G. B., 27 aoûं 1960, 187 (4739) : 778.

2. BURSELL ( $E$.). - Aspects of the metabolism of amino acids in the tse tse fly glossina (Diptera). J. insect. physiol., 1963, $9: 439-52$.

3. BURSELL (E.). - Oxaloacéticcarboxylase in flight musculature of the tse tse fly. Comp. Biochem. physiol., 1965, $16: 259-66$.

4. GEIGY (R.), HUBER (M.), WEINMAN (D.) et WYATT (G. R.). - Demonstration of trehalose in the vector of African trypanosomiasis: the tse tse fly. Acta Trop., 1959, 16 (3) : 255-62.

5. GRACE (T.D. C.). - The prolonged growth and survival of ovarian tissues of the promethea moth (Callosamia promethea) in vitro. J. Gen. Physiol., 1958, $41: 1027$.

6. GRACE (T, D. C.). - Effects of various substances on growth of silkworm tissues in vitro. Aust. J. biol. Scien., 1958, II : 407.
7. ITARD (J.). - Rapport annuel du laboratoire d'Entomologie de la portion centrale de I'I. E. M. V. T., 1967.

8. KNIGHT (R. H.). - Free amino-acids in the haemolymph of Glossina species. E.A.T.R.O. Report Journ. Déc. 1960, publié en 1961. p. 22-23.

9. NICOLI (J.) et VATTIER (G.). - Culture de Trypanosoma rhodesense sur tissus de pupes de glossines. Bull. Soc. poth. exot, 1964. 57 (2) : 213-19.

10. TRAGER (W.). - Tse tse fly tissue culture and the development of trypanosomes to the infective stage. Ann. trop. Med. Parasit., 1959, $53: 473-91$.

11. WYATT (S. S.). - Culture in vifro of tissue from the silkworm Bombyx mori L. J. Gen. Physiol., 1956, 39 : 841. 